\title{
Tarifa socialmente justa para el servicio de transporte público urbano y su relación con los tiempos de recorrido e intervalos de despacho
}

\section{Socially fair rate for urban public transport service and its relation to travel times and dispatch intervals}

Wilson E. Jaramillo S.

Universidad Internacional del Ecuador, Ecuador

Luis R. Jácome G.

Universidad Nacional de Loja, Ecuador

Autor para correspondencia: wijaramillosa@uide.edu.ec

Fecha de recepción: 10 de septiembre de 2017 - Fecha de aceptación: 30 de noviembre de 2017

Resumen: Definir una tarifa socialmente justa para el servicio de transporte público es un tema de importancia que preocupa a las autoridades que regulan la movilidad en las ciudades. En la presente investigación se analiza la relación que existe entre la velocidad de recorrido de los vehículos, con el valor de tarifa necesaria para lograr el punto de equilibrio entre ingresos y gastos. El método de investigación empleado contempla el análisis de varios escenarios de operación. Para el análisis de cada escenario se emplea como herramienta informática el software ITP, el cual genera información que permite el cálculo de la tarifa empleando la metodología definida por la autoridad Nacional de Tránsito. Los procedimientos empleados para el desarrollo de la investigación pueden ser replicados, concluyendo finalmente que a mayor velocidad de recorrido los costos operativos de la ruta disminuyen, lo que se traduce en un menor valor de la tarifa.

Palabras clave: tarifa; transporte público; buses; demanda; costos operativos; velocidad; variación horaria de la demanda; intervalo de despacho

\begin{abstract}
Define a socially fair rate for public transportation is an important issue that concerns the authorities to regulate the mobility in cities. In the present investigation the relationship between the speed of vehicle travel with the value of rate necessary to achieve the balance between revenue and expenditure. The research method employed includes the analysis of various operating scenarios. For the analysis of each scenario is used as the ITP software tool, which generates information that allows the calculation of the rate using the methodology defined by the ANT Ecuador. The procedures used for the development of research can be replicated in all kinds of routes andany city, finally concluding that the higher the travel speed, the lower the operating costs of the route.
\end{abstract}

Key words: rate; public transportation; buses; demand; operating costs; speed; hourly variation in demand 


\section{Introducción}

En el año 2014 en el Ecuador, el tema de la tarifa del servicio de transporte público se ha convertido en uno de los principales temas de discusión y análisis político, debido al alto grado de sensibilidad social que conlleva decidir sobre el tema. Por un lado, las operadoras de transporte público solicitan a las autoridades el alza de pasajes dado el incremento en los últimos años de los costos de operación y mantenimiento de las unidades como se expone en la Revista Buen Viaje (Nro. 113, 2014) y por otro la población solicita se mejore la seguridad y calidad del servicio.

Desde el punto de vista económico, el valor de la tarifa responde a un equilibrio entre los costos de operación y mantenimiento del sistema de transporte público (oferta) y los ingresos generados producto del pago realizado por los usuarios del servicio (Demanda).

Con respecto a los costos operativos Molinero (1997) manifiesta que la cantidad de vehículos es el parámetro que mejor refleja el equilibrio entre la oferta y la demanda del transporte colectivo. Así, por ejemplo, en el caso de contar con más autobuses en servicio que los requeridos, se produce un exceso de oferta y una ociosidad del equipo, lo cual conduce a un alto costo en la operación que invariablemente se traduce en tarifas excesivas para el usuario. Por otra parte, si se cuenta con un parque vehicular por abajo de los requerimientos reales, se presenta una mala calidad del servicio que se traduce en molestias e inseguridad al usuario.

Para garantizar un nivel adecuado de servicio existen técnicas de programación que basadas en datos de campo permiten generar itinerarios de operación para cada ruta y vehículo, siendo necesario para su aplicación conocer las siguientes definiciones presentadas por Molinero (1997):

Intervalo de despacho: El intervalo es la porción de tiempo, comúnmente expresada en minutos, entre dos salidas sucesivas de vehículos de transporte público en una ruta.

Frecuencia: La frecuencia es el número de unidades que pasan un punto dado en la ruta durante una hora (o cualquier periodo de tiempo considerado).

Capacidad vehicular: La capacidad vehicular es el número total de espacios en el vehículo tanto de pie como sentados.

Volumen de pasajeros: El volumen de pasajeros es el número de usuarios que pasan por un punto fijo durante una hora. El volumen de pasajeros varía conforme las variaciones de la hora del día, de la semana y época del año.

Sección de máxima demanda: La sección de máxima demanda es el punto dentro de la ruta donde se tiene el mayor número de usuarios abordo y establece el volumen de diseño de la ruta.

Volumen de diseño: El volumen de diseño es el que se presenta en la sección de máxima demanda de una ruta, y, en consecuencia, el mayor volumen de cualquier parada o sección a lo 
largo de la ruta. Este volumen es el parámetro básico para determinar la capacidad de línea que debe ofrecerse.

Capacidad de línea ofrecida: La capacidad de la ruta es el número total de espacios ofrecidos en un punto de una ruta durante una hora y es resultado del producto de la frecuencia y la capacidad vehicular.

Tiempo de recorrido: Es el tiempo que emplea un vehículo de una terminal (cierre de circuito) a la terminal opuesta en una ruta. El tiempo de recorrido se expresa usualmente en minutos.

Factor de ocupación: Resulta de dividir el número de usuarios a bordo de una unidad entre la capacidad vehicular.

Tiempo de terminal: Es el tiempo entre que llega y vuelve a salir un vehículo de la terminal o cierre de circuito. Su propósito es contar con tiempo para dar la vuelta al vehículo, para dar un descanso al operador y, para permitir los ajustes necesarios en el horario.

Tiempo de ciclo o vuelta: Es el tiempo total de viaje redondo para una unidad de transporte, esto es, el tiempo que tarda en volver a pasar la misma unidad por un punto determinado.

Velocidad de recorrido: La velocidad de recorrido es producto de la relación entre el tiempo que emplea un vehículo en recorrer una ruta desde un terminal a otro opuesto por la longitud de recorrido. La velocidad de recorrido se expresa usualmente en kilómetros por hora. Es importante aclarar que esta velocidad incluye las demoras para recoger y dejar pasajeros a lo largo de la ruta y otras demoras producto del tráfico vehicular.

Tamaño del parque vehicular: es el número total de unidades que operan en una ruta y con el que se atiende adecuadamente la demanda de pasajeros.

Rutas diametrales: Se caracterizan por tener dos terminales de despacho de vehículos, en cada extremo de la ruta.

Rutas radiales: Se caracterizan por tener un solo terminal de despacho de vehículos.

Con la información necesaria y aplicando los conceptos antes expuestos se puede realizar una programación adecuada que garantice un nivel de servicio de transporte público que agrade y satisfaga la necesidad de los usuarios, existiendo para esto pocas herramientas informáticas especializadas como el software llamado ITP (Jaramillo, 2012).

El software ITP (Enlace al video: http://youtu.be/vQUIRC7Ax8?list=UUSUUVLmLYLHCjM6nSalPJQA) permite programar la operación del servicio de transporte público, para lo cual el usuario de la herramienta informática ingresa intervalos de despacho, longitudes y tiempos de recorrido, capacidad del vehículo y factor de ocupación, obteniendo como resultado para horas valle y horas pico la capacidad en línea de la ruta y el número requerido de buses. Para cada bus de la ruta se genera un itinerario de operación que detalla el número de vueltas, kilómetros a recorrer por día y el tiempo de servicio del conductor. 
La información obtenida permite además realizar el cálculo de los costos fijos y variables de operación y mantenimiento de los vehículos considerando para esto la vida útil, inversión, financiamiento, costo de repuestos, combustible, llantas y otros insumos necesarios para el funcionamiento del sistema de transporte público. Relacionar el costo operativo con la demanda de pasajeros de la ruta genera como resultado el valor de la tarifa de equilibrio.

Retomando nuevamente el tema de la calidad y el adecuado nivel de servicio, se considera el criterio de Moller (2009) quien, al tratar sobre la reorganización del transporte público colectivo en ciudades de tamaño medio, manifiesta, importante para la calidad del transporte público colectivo es su velocidad; el objetivo debe ser llegar dentro de la zona urbana a una velocidad promedio de por lo menos $20 \mathrm{Km} / \mathrm{h}$ - mejor sería $25 \mathrm{Km} / \mathrm{h}$.

Con respecto a las características de operación de las rutas de transporte pública en ciudades de tamaño medio se toma como referencia a la ciudad de Loja, en donde de conformidad a información transmitida por funcionarios de la Unidad Municipal de Transporte Terrestre, Tránsito y Seguridad Vial, la velocidad de recorrido de las unidades de transporte público en las distintas rutas oscila entre 14 y $18 \mathrm{Km} / \mathrm{h}$.Así mismo, una característica que presentan las rutas de la ciudad es que se evidencia a lo largo del día variación de la demanda en donde se puede determinar las horas de mayor demanda u horas pico y las de menor demanda $\mathrm{u}$ horas valle.

En el marco de la movilidad sostenible de las ciudades lograr un nivel adecuado de servicio de transporte público con una tarifa socialmente justa, un menor consumo de energía e impacto ambiental, de seguro que beneficia a la población, lo que motiva a que el objetivo de la presente investigación sea: Conocer la relación existente entre el costo de la tarifa y la velocidad de recorrido del servicio de transporte público, considerando la variación de intervalos de despacho.

Las preguntas de investigación son:

$\checkmark \quad$ ¿La velocidad de recorrido de las unidades de transporte público incide en el costo de la tarifa?

$\checkmark$ ¿Al considerar la variación de intervalos en relación a la demanda existente en las horas de mayor y menor demanda, se puede disminuir los costos de operación del sistema y por ende el valor de la tarifa?

Las hipótesis que orientan la investigación son:

- A mayor velocidad de operación en una ruta, disminuye el valor de la tarifa.

- La tarifa del sistema de transporte público disminuye al definir distintos intervalos de despacho, de conformidad al flujo de pasajeros existente en las horas de menor y mayor demanda.

\section{Materiales y métodos}


Con el propósito de responder a las preguntas de investigación planteadas y cumplir con el objetivo de estudio, se somete a prueba las hipótesis usando el software ITP, herramienta informática que se utiliza para simular la operación de rutas de transporte público, para lo cual en el diseño de investigación se considera como variable independiente a la velocidad de operación y como variable dependiente el valor de la tarifa necesaria para obtener un equilibrio entre costos e ingresos.

Para dar respuesta a la primera pregunta de investigación y dar validez a los resultados a través de la equivalencia de escenarios, se considera el siguiente supuesto para cada simulación:

a) Se supone una ruta diametral cualquiera cuya longitud de recorrido es de $12 \mathrm{Km}$ en el sentido Norte - Sur y de $12 \mathrm{Km}$ en el sentido contrario, es decir un total de recorrido por vuelta de $24 \mathrm{Km}$.

b) La demanda promedio de la ruta es de 30000 viajes/día.

c) El intervalo de despacho de la rutaes de 3 minutos y se mantiene constante durante el periodo de operación que es desde las 06h00 hasta las $21 \mathrm{~h} 00$.

d) El tiempo total de terminal o de descanso de la unidad por cada vuelta es de 20 minutos.

e) La variable independiente es la velocidad de recorrido de las unidades, para lo cual se manipula intencionalmente los tiempos de recorrido de la ruta con la finalidad de obtener velocidades de 24, 22, 20, 18, 16 y $14 \mathrm{Km} / \mathrm{h}$.

f) Se considera que la capacidad de los buses es de 75 pasajeros y el factor de ocupación de 0.9 .

g) Con el valor de cada variable independiente se calcula el número de vehículos requerido, la cantidad de kilómetros a recorrer y los costos de operación y mantenimiento, lo que nos permite definir el valor de la tarifa de equilibrio (variable dependiente) relacionando los costos con la demanda de pasajeros de la ruta.

h) Para el cálculo de la tarifa se emplea la Metodología para la fijación de tarifas de transporte terrestre intracantonal o urbano, definida por la Agencia Nacional de Transporte Terrestre, Tránsito y Seguridad Vial del Ecuador mediante resolución Nro. 122-DIR-ANT-2014.

Para dar respuesta a la segunda pregunta de investigación se considera los mismos supuestos anteriores, solo que se modifica el supuesto del literal c), por el siguiente:

i) Se supone que en vez de despachar durante todo el día las unidades de transporte público a un intervalo constante de 3 minutos se considera la variación horaria de la demanda (horas pico y horas valle), con intervalos de despacho de 3, 4 y 5 minutos de conformidad al siguiente cuadro:

\begin{tabular}{cc}
\hline Periodo & $\begin{array}{c}\text { Intervalo de } \\
\text { despacho }\end{array}$ \\
\hline 06h00 a 08h30 & 3 \\
08h30 a 11h30 & 5 \\
11h30 a $15 h 30$ & 3 \\
15h30 a $17 \mathrm{~h} 30$ & 5 \\
17h30 a $19 \mathrm{~h} 30$ & 3 \\
19h30 a $21 \mathrm{~h} 00$ & 4 \\
\hline
\end{tabular}


Cuadro 1. Intervalos de despacho considerando variación de la demanda Elaborado por: Autor de la investigación

\section{Resultados}

Los resultados de investigación demuestran que existe variación en el valor de la tarifa en relación a la velocidad de operación, tarifa que inclusive puede disminuir al considerar durante la operación del servicio de transporte público, la variación en los tiempos de intervalos de despacho. En el gráfico 1 siguiente se detallan los resultados.

Como se puede observar en la tabla que forma parte del gráfico 1, cuando la velocidad de recorrido de las unidades es mayor se requiere un menor número de unidades para atender la demanda existente en la ruta, lo que se refleja en menores costos de operación y mantenimiento y consecutivamente en un valor menor de la tarifa de equilibrio.

En el cuadro 2 se presenta el porcentaje de variación de la tarifa tomando como referencia el valor obtenido en el escenario cuya velocidad de recorrido es de $24 \mathrm{Km} / \mathrm{h}$, se puede apreciar que al disminuir la velocidad de recorrido de 24 a $14 \mathrm{Km} / \mathrm{h}$ la tarifa se incrementa hasta en un $23.99 \%$, lo que responde a la primera pregunta de investigación y comprueba la primera hipótesis.

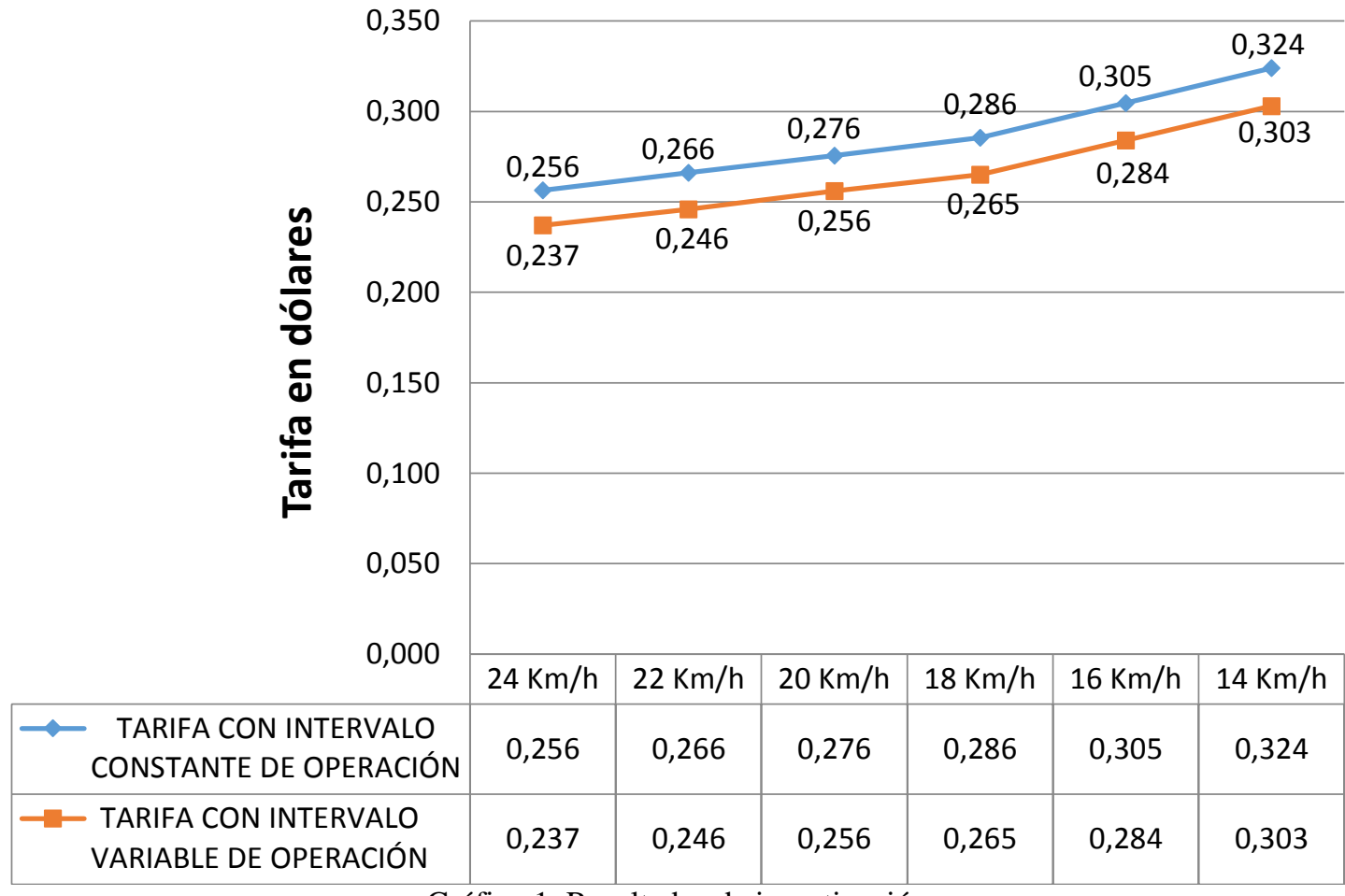

Gráfico 1. Resultados de investigación

\begin{tabular}{ccccccc}
\hline Pasajero/Bus/Día & 1071 & 1000 & 938 & 883 & 790 & 715 \\
\hline $\begin{array}{c}\text { Prom. Km/día/bus recorridos } \\
\text { Intervalo constante hp }\end{array}$ & 257.14 & 240.00 & 225.00 & 211.76 & 189.47 & 171.43 \\
$\begin{array}{c}\text { Prom. Km/día/bus recorridos } \\
\text { Intervalo variable hp y hv }\end{array}$ & 216.86 & 202.40 & 189.75 & 178.59 & 159.79 & 144.57 \\
\hline
\end{tabular}




\begin{tabular}{ccccccc}
\hline $\begin{array}{c}\text { Nro. de autobuses requerido } \\
\text { Oferta de km/día int. }\end{array}$ & 28 & 30 & 32 & 34 & 38 & 42 \\
$\quad$ Constante & & 7200 & 7200 & 7200 & 7200 & 7200 \\
Oferta de km/día int. & 6072 & 6072 & 6072 & 6072 & 6072 & 6072 \\
Variable & & & & & & \\
Velocidad de recorrido & 24 & 22 & 20 & 18 & 16 & 14 \\
& $\mathrm{Km} / \mathrm{h}$ & $\mathrm{Km} / \mathrm{h}$ & $\mathrm{Km} / \mathrm{h}$ & $\mathrm{Km} / \mathrm{h}$ & $\mathrm{Km} / \mathrm{h}$ & $\mathrm{Km} / \mathrm{h}$ \\
Tiempo de terminal & 20 & 20 & 20 & 20 & 20 & 20 \\
& $\mathrm{~min}$ & $\mathrm{~min}$ & $\mathrm{~min}$ & $\mathrm{~min}$ & $\mathrm{~min}$ & $\mathrm{~min}$ \\
Tiempo de recorrido & 60 & 66 & 72 & 82 & 92 & 104 \\
& $\mathrm{~min}$ & $\mathrm{~min}$ & $\mathrm{~min}$ & $\mathrm{~min}$ & $\mathrm{~min}$ & $\mathrm{~min}$ \\
Longitud de recorrido & $24 \mathrm{Km}$ & $24 \mathrm{Km}$ & $24 \mathrm{Km}$ & $24 \mathrm{Km}$ & $24 \mathrm{Km}$ & $24 \mathrm{Km}$ \\
Capacidad en línea & 1350 & 1350 & 1350 & 1350 & 1350 & 1350 \\
pasajero/hp & & & & & & \\
Capacidad en línea & 810 & 810 & 810 & 810 & 810 & 810 \\
pasajero/hv & & & & & & \\
Intervalo hora pico (hp) & $3 \mathrm{~min}$ & $3 \mathrm{~min}$ & $3 \mathrm{~min}$ & $3 \mathrm{~min}$ & $3 \mathrm{~min}$ & $3 \mathrm{~min}$ \\
Intervalo hora valle (hv) & $5 \mathrm{~min}$ & $5 \mathrm{~min}$ & $5 \mathrm{~min}$ & $5 \mathrm{~min}$ & $5 \mathrm{~min}$ & $5 \mathrm{~min}$ \\
Factor de ocupación & 0.9 & 0.9 & 0.9 & 0.9 & 0.9 & 0.9 \\
Capacidad del vehículo pasaj. & 75 & 75 & 75 & 75 & 75 & 75 \\
\hline
\end{tabular}

\begin{tabular}{cccc}
\hline & Tarifa & \% Incremento & \% Incremento \\
\hline Velocidad & Dólar & Tarifa & Tarifa \\
Variable Independiente & Variable Dependiente & Parcial & Acumulada \\
$24 \mathrm{Km} / \mathrm{H}$ & 0.256 & $0.00 \%$ & $0.00 \%$ \\
$22 \mathrm{Km} / \mathrm{H}$ & 0.266 & $3.79 \%$ & $3.79 \%$ \\
$20 \mathrm{Km} / \mathrm{H}$ & 0.276 & $3.56 \%$ & $7.35 \%$ \\
$18 \mathrm{Km} / \mathrm{H}$ & 0.286 & $3.62 \%$ & $10.97 \%$ \\
$16 \mathrm{Km} / \mathrm{H}$ & 0.305 & $6.72 \%$ & $17.69 \%$ \\
$14 \mathrm{Km} / \mathrm{H}$ & 0.324 & $6.30 \%$ & $23.99 \%$ \\
\hline
\end{tabular}

Cuadro 2. Porcentaje de variación de la tarifa con relación a la velocidad de operación manteniendo un intervalo de despacho constante

Elaborado por: Autor de la investigación

Al considerar para los mismos escenarios de investigación, la variación de los intervalos de despacho de las unidades durante el día de operación, los kilómetros que deben recorrer las unidades para atender las necesidades de la demanda disminuye en un $18.58 \%$, como se puede observar en el cuadro 3.

\begin{tabular}{|c|c|c|c|c|c|c|}
\hline & \multicolumn{2}{|c|}{ Intervalo Constante } & \multicolumn{2}{|c|}{ Intervalo Variable } & Diferencia & $\%$ \\
\hline $\begin{array}{c}\text { Velocidad } \\
\text { Operación } \\
\mathrm{Km} / \mathrm{h}\end{array}$ & $\begin{array}{l}\text { Número de } \\
\text { buses } \\
\text { requerido }\end{array}$ & $\begin{array}{c}\text { Kilómetros } \\
\text { recorridos } \\
\text { Bus/día }(\mathrm{KC})\end{array}$ & $\begin{array}{l}\text { Número de } \\
\text { buses } \\
\text { requerido }\end{array}$ & $\begin{array}{c}\text { Kilómetros } \\
\text { recorridos } \\
\text { Bus/día }(\mathrm{KV})\end{array}$ & $\begin{array}{c}\text { Kilómetros } \\
\text { recorridos } \\
\mathrm{KD}=\mathrm{KC}-\mathrm{KV}\end{array}$ & $\begin{array}{c}\text { Disminución } \\
\text { Kilómetros } \\
\text { recorridos }\end{array}$ \\
\hline $24 \mathrm{Km} / \mathrm{h}$ & 28 & 257.14 & 28 & 216.86 & 40.29 & $18.58 \%$ \\
\hline $22 \mathrm{Km} / \mathrm{h}$ & 30 & 240.00 & 30 & 202.40 & 37.60 & $18.58 \%$ \\
\hline $20 \mathrm{Km} / \mathrm{h}$ & 32 & 225.00 & 32 & 189.75 & 35.25 & $18.58 \%$ \\
\hline $18 \mathrm{Km} / \mathrm{h}$ & 34 & 211.76 & 34 & 178.59 & 33.18 & $18.58 \%$ \\
\hline $16 \mathrm{Km} / \mathrm{h}$ & 38 & 189.47 & 38 & 159.79 & 29.68 & $18.58 \%$ \\
\hline $14 \mathrm{Km} / \mathrm{h}$ & 42 & 171.43 & 42 & 144.57 & 26.86 & $18.58 \%$ \\
\hline
\end{tabular}

Cuadro 3. Comparación de los resultados de número de unidades y kilómetros recorridos para cada escenario de investigación, considerando intervalos contantes y variables de despacho 
Elaboración: Autor de la investigación

Al disminuir la cantidad de kilómetros recorridos por cada vehículo, también disminuye el costo de operación y mantenimiento de los mismos, lo que provoca una disminución en el valor de la tarifa en un rango que oscila entre el $6.9 \%$ al $8.18 \%$, como se puede observar en el cuadro 4.

\begin{tabular}{ccccc}
\hline $\begin{array}{c}\text { Velocidad } \\
\text { Operación }\end{array}$ & $\begin{array}{c}\text { Intervalo } \\
\text { Constante } \\
\text { Tarifa (A) }\end{array}$ & $\begin{array}{c}\text { Intervalo } \\
\text { Variable } \\
\text { Tarifa (B) }\end{array}$ & $\begin{array}{c}\text { Diferencia } \\
\text { De Tarifas } \\
\text { C=A-B }\end{array}$ & $\begin{array}{c}\% \\
\text { Disminución } \\
\text { Tarifa }\end{array}$ \\
\hline $24 \mathrm{Km} / \mathrm{H}$ & 0.256 & 0.237 & 0.019 & $8.18 \%$ \\
$22 \mathrm{Km} / \mathrm{H}$ & 0.266 & 0.246 & 0.020 & $8.18 \%$ \\
$20 \mathrm{Km} / \mathrm{H}$ & 0.276 & 0.256 & 0.020 & $7.65 \%$ \\
$18 \mathrm{Km} / \mathrm{H}$ & 0.286 & 0.265 & 0.021 & $7.75 \%$ \\
$16 \mathrm{Km} / \mathrm{H}$ & 0.305 & 0.284 & 0.021 & $7.30 \%$ \\
$14 \mathrm{Km} / \mathrm{H}$ & 0.324 & 0.303 & 0.021 & $6.90 \%$ \\
\hline
\end{tabular}

Cuadro 4. Comparación de los resultados del valor de la tarifa para cada escenario de investigación considerando intervalos constantes y variables de despacho

Elaboración: Autor de la investigación

Los resultados del cuadro anterior responden a la segunda pregunta de investigación comprobándose además la segunda hipótesis.

\section{Discusión}

Los resultados del estudio dejan en claro que el tema de la tarifa del servicio de transporte público debe ser manejado de manera técnica, considerando parámetros como la velocidad de operación y la variación existente de demanda en el transcurso de los diferentes días de la semana, es decir debe existir un itinerario de despacho de unidades para días ordinarios, sábados, domingo y feriados.

Con respecto a la velocidad de recorrido los resultados de la investigación demuestran que es necesario que las operadoras de transporte público y las autoridades encargadas de la planificación, regulación y control del servicio, concentren esfuerzos para eliminar las demoras innecesarias en los recorridos de cada ruta, se optimice los tiempos de recorrido y se aumente las velocidades de recorrido, en lo posible en un rango que oscile de 20 a $25 \mathrm{Km} / \mathrm{h}$. De esta manera se puede atender la demanda con un nivel de servicio adecuado, generando un menor costo operativo, lo que al final se traduce en un valor menor de la tarifa.

Los resultados concuerdan con lo expuesto en la literatura considerada en la elaboración de la presente investigación, especialmente en las referencias realizadas a Molinero (1997) quien relaciona la sobreoferta de servicio y la ociosidad de vehículos con el valor excesivo de la tarifa y Moller (2009) quien considera que la velocidad de recorrido tiene incidencia en un adecuado nivel de servicio.

Es necesario dejar en claro que cuando se trata sobre la velocidad de recorrido, no se está relacionado el tema con los correteos y aguantes que ocurren en la actualidad en muchas rutas de 
transporte público del Ecuador, producto de la llamada guerra del centavo que aumenta los costos operativos y disminuye la vida útil de las unidades de transporte, y lo que es peor, pone en riesgo la vida de usuarios y transeúntes.

Los procedimientos realizados para efectuar la presente investigación tienen aplicación práctica y pueden ser replicados para definir la tarifa en rutas de transporte público de cualquier ciudad del mundo, únicamente se requiere contar con la herramienta informática que permita la programación y elaboración de itinerarios para cada ruta, como es el software ITP, datos operativos y de campo de cada ruta y el valor actualizado de los insumos(lubricantes, repuestos, llantas, etc.) que inciden en la operación y mantenimiento de los vehículos.

\section{Conclusión}

Se cumple con el objetivo de investigación ya que los resultados permiten conocer la relación que existe entre la velocidad de recorrido de los buses de transporte urbano con el valor necesario de tarifa para mantener un equilibrio entre costos e ingresos del sistema. A mayor velocidad de recorrido es menor el valor de la tarifa a pagar por el usuario.

Para los escenarios considerados en la presente investigación se puede apreciar que al disminuir la velocidad de recorrido de 24 a $14 \mathrm{Km} / \mathrm{h}$, aumentan los costos de operación y mantenimiento de la ruta, por lo que la tarifa se incrementa hasta en un $23.99 \%$.

Definir distintos intervalos de despacho de conformidad a la demanda existente en horas de máxima y menor demanda, permite optimizar el costo de operación y mantenimiento de la ruta, ya que disminuye la cantidad de kilómetros rodados por cada vehículo, para el caso de la presente investigación disminuye en un $18.58 \%$.

Para el presente estudio el disminuir la cantidad de kilómetros recorridos por cada vehículo sin afectar los niveles de servicio relacionados a la capacidad de los vehículos para transportar pasajeros, genera para los distintos escenarios de análisis una disminución del valor de la tarifa en un rango que oscila entre el $6.9 \%$ al $8.18 \%$.

Los procedimientos aplicados en el presente estudio contribuyen a la búsqueda de una tarifa de servicio de transporte público socialmente justa, lo que puede ser replicado para la definición de la tarifa en las rutas de cualquier ciudad del mundo.

\section{Reconocimiento}

Mi agradecimiento a la Universidad Internacional del Ecuador extensión Loja, en especial a la Arq. Tatiana Trokhimtchouk Directora del CipArq-Loja, por dar las facilidades para elaborar el presente trabajo de investigación, por el tiempo invertido y los comentarios generados con respecto al tema, así mismo a funcionarios del Municipio de Loja.

\section{Bibliografía}


ANT (2014). Resolución de la Agencia Nacional de Transporte Terrestre, Tránsito y Seguridad Vial Nro. 122-DIR-ANT-2014, Metodología para la fijación de tarifas de transporte terrestre intracantonal o urbano. Quito, 03 de octubre de 2014.

Asamblea Constituyente (2008). Constitución del Ecuador. Registro oficial del 20 de octubre de 2008. Ecuador.

Asamblea Nacional del Ecuador (2008). Ley Orgánica de Transporte Terrestre, Tránsito y Seguridad Vial. Registro Oficial Nro. 398 del 07 de agosto del 2008. Ecuador.

Asamblea Nacional del Ecuador (2010). Código Orgánico de Organización Territorial Autonomía y Descentralización. Registro Oficial Nro. 303 del 19 de octubre del 2010. Ecuador.

Asamblea Nacional del Ecuador (2014). Código Orgánico Integral Penal. Registro Oficial Nro. 180 del lunes 10 de febrero del 2014. Ecuador.

Hernández, R. (2010). Metodología de la Investigación. MacGraw Hill, Quinta edición México. 613 pág.

Jaramillo, W. y Jácome, L. (2012). Software ITP "Itinerarios para rutas de transporte público". Programa de Ordenador. Certificado IEPI Nro. CUE -000866. Loja - Ecuador.

Jiménez, C. (2015). Educación Vial "Hacia el tránsito seguro y sostenible”. España. McGraw Hill. Recuperado de: ProQuestebrary. Web.

Lizárraga, C. (2006). Movilidad urbana sostenible: un reto para las ciudades del siglo XXI. Economía, Sociedad y Territorio, vol. VI, núm. 22. Facultad de Ciencias Económicas y Empresariales, Universidad de Granada. España.

Molinero, A. (1997). Transporte Público: Planeación, Diseño, Operación y Administración. Universidad Autónoma del Estado de México, 776 pág.

Moller R. (2006). Transporte Urbano y Desarrollo Sostenible en América Latina. Universidad del Valle. Cali - Colombia. 166 pág.

Moller R. (2009). Transporte Urbano Sostenible y Calidad de Vida para los Municipios de Colombia. Universidad del Valle. Cali - Colombia. 166 pág.

Municipio de Loja (2012). Plan de Movilidad del Cantón Loja 2012 - 2022. Ecuador.

Municipio de Loja (2014). Plan de Desarrollo y Ordenamiento Territorial del Cantón Loja 2014-2022. Ecuador.

Revista Buen Viaje (2014). edición 113 de octubre - noviembre 2014. Quito - Ecuador. 